AIAA 2002-2757

Optimal Control of Aeroacoustic

Flows: Transpiration Boundary

Control

S. Scott Collis, Kaveh Ghayour,

and Matthias Heinkenschloss

Rice University

Houston, Texas 77005-1892

3rd AIAA Theoretical Fluid Mechanics

Meeting

June 24-27, 2002

St. Louis, Missouri 


\title{
Optimal Control of Aeroacoustic Flows: Transpiration Boundary Control
}

\author{
S. Scott Collis, ${ }^{*}$ Kaveh Ghayour, ${ }^{\dagger}$ \\ and Matthias Heinkenschloss ${ }^{\ddagger}$ \\ Rice University \\ Houston, Texas 77005-1892
}

\begin{abstract}
We consider the optimal boundary control of aeroacoustic noise governed by the two-dimensional unsteady compressible Euler equations. The control is the time and space varying wall-normal velocity (transpiration velocity) on a subset of a solid wall. The objective functional to be minimized is a measure of acoustic amplitude. Optimal transpiration boundary control of aeroacoustic noise introduces challenges beyond those encountered in direct aeroacoustic simulations or in many other optimization problems governed by compressible Euler equations. One nontrivial issue that arises in our optimal control problem is the formulation and implementation of transpiration boundary conditions. Since we allow suction and blowing on the boundary, portions of the boundary may change from inflow to outflow, or vice versa, and different numbers of boundary conditions must be imposed depending on whether a boundary portion is an inflow or an outflow boundary. Another important issue is the derivation of adjoint equations, which are needed for the computation of the gradient of the objective function with respect to the control. Among other things, this is influenced by the choice of boundary conditions for the compressible Euler equations. This paper describes our approaches to meet these challenges and presents first results for two model problems. These problems are designed to validate our transpiration boundary conditions and their implementation, study the accuracy of gradient computations, and assess the performance of the computed controls.
\end{abstract}

\section{Introduction}

The goals of this paper are the description of issues arising in the computation of optimal transpiration boundary control for the minimization of aeroacoustic noise, the introduction of our approaches to deal with these issues, and the presentation of first results that support the effectiveness of our approach.

The control objective for aeroacoustic applications usually targets acoustic waves that are typically several orders of magnitude smaller than flow quantities associated with the energetically dominant dynamics. Like the direct simulation of aeroacoustic phenomena, optimal control of aeroacoustics requires proper resolution of small amplitude acoustic fluctuations. However, optimal control imposes additional demands. First, optimal control of aeroacoustic noise requires that dynamic control actuations are properly translated into small amplitude acoustic fluctuations. This can add significant challenges to the simulation. Secondly, to apply gradient based optimization algorithms for the computation of an optimal control, one needs to compute sensitivity information. In our case, where controls are temporally and spatially distributed, this is accomplished

*Assistant Professor, Department of Mechanical Engineering and Materials Science, collis@ rice.edu, member AIAA.

${ }^{\dagger}$ Postdoctoral Associate, Department of Computational and Applied Mathematics and Department of Mechanical Engineering and Materials Science, kghayour@caam.rice.edu

$¥$ Associate Professor, Department of Computational and Applied Mathematics, heinken@ rice.edu

Copyright (c) 2002 by the authors. Published by the American Institute of Aeronautics and Astronautics, Inc. with permission. using the adjoint equation approach. The implementation of the adjoint equation needs to accurately relate undesired small amplitude acoustic fluctuations to modifications of the control that can reduce them.

Acoustic waves are non-dissipative and non-dispersive; their resolution requires high-order accurate numerical schemes with minimal dissipation and dispersion error. In our state computations, spatial derivative operators are discretized with sixth-order central finite-differences and a fourth-order accurate explicit Runge-Kutta scheme is used to advance the solution in time. We use sponge regions near the far field boundaries to allow acoustic waves and spurious numerical waves leave the domain without significant reflections. A nonstandard and nontrivial task that arises in our optimal control problem is the formulation and implementation of transpiration boundary conditions. Since we allow suction and blowing on the boundary, portions of the boundary are allowed to change dynamically from inflow to outflow, or vice versa. Different numbers of boundary conditions have to be imposed depending on whether a boundary portion is an inflow or an outflow boundary. Specifically, for subsonic suction only one physical boundary condition is required while three physical boundary conditions are needed for subsonic blowing. Our choice of boundary conditions as well as their implementation in the context of our high-order finite-difference based code is discussed below. Our implementation of the boundary conditions borrows heavily from Sesterhen. ${ }^{1}$

Since the number of control variables in our problem is 
large, we apply a gradient based algorithm for the computation of an optimal control. The gradient of the objective function with respect to the control is computed using the adjoint equation approach. The general framework underlying our approach in this paper is the same as that used in our previous work ${ }^{2,3}$ and it is similar to many adjoint equation based optimization procedures used for optimization of unsteady problems, although it carefully addresses some subtle but important issues that are frequently overlooked in other work. In our prior work ${ }^{2}$ on the optimal boundary control of unsteady, two-dimensional compressible flows, we used the discretize-then-optimize approach for gradient computation. In this approach the state equation and objective function are first discretized and the adjoint calculus is applied to the discrete problem, possibly aided by automatic differentiation. While this method generates exact gradient information for the discretized problem, it does so without providing direct physical and mathematical insight into the adjoint partial differential equations, especially their boundary conditions, associated with the continuous problem. The latter are important to assess the well-posedness of the optimal control problem and the quality of the computed discretized control as an approximation to the true optimal control. Accurate state discretizations are not sufficient to ensure an accurate discretization of the optimal control problem. For the latter it is also necessary to adequately resolve the adjoint partial differential equations. Gradient computations using the optimize-then-discretize approach, in which one first determines the adjoint partial differential equations and their boundary conditions and then discretizes these, in combination with the aforementioned discretize-then-optimize approach can provide some insight into the issues of wellposedness and approximation quality. Although study of these issues is beyond the scope of this paper, it is part of our overall goals. Therefore our procedure in this paper is guided by the optimize-then-discretize approach.

In order to test our approach, two optimal control problems are solved and discussed in this paper. The first test problem shows that the computed optimal controls can produce well-resolved planar acoustic waves of very small amplitude to minimize the observed sound amplitudes by means of wave-cancellation. In the second test problem, the optimal control targets the no-penetration solid wall boundary condition which amplifies the noise amplitude by reflecting the incident waves back into the observation region. The computed optimal control lets the incident acoustic waves pass through the wall, mimicking a nonreflecting boundary condition.

\section{Problem Formulation}

\section{State equations}

The Euler equations can be written in conservative form as

$$
\mathbf{q}(\mathbf{u})_{t}+\sum_{i=1}^{2} \mathbf{F}^{i}(\mathbf{u})_{x_{i}}=\mathbf{0} \quad \text { in }\left(t_{0}, t_{f}\right) \times \Omega
$$

with initial condition $\mathbf{u}\left(t_{0}, \mathbf{x}\right)=\mathbf{u}_{0}(\mathbf{x})$ and boundary conditions $\mathbf{B}(\mathbf{u}, \nabla \mathbf{u}, g)=\mathbf{0}$. In $(1), \mathbf{u}=\left(\rho, v_{1}, v_{2}, T\right)^{T}$ are the primitive variables and $\mathbf{q}(\mathbf{u})=\left(\rho, \rho v_{1}, \rho v_{2}, \rho E\right)^{T}$ are the conservative variables, using the common notation of $\rho, v_{i}$ and $T$ for density, velocity component in $x_{i}$-direction, and temperature, respectively. $\mathbf{F}^{i}$ denotes the convective flux in the $x_{i}$ direction and the pressure $p$ and total energy per unit mass $E$ are given by

$$
p=\frac{\rho T}{\gamma \mathrm{M}^{2}} \quad E=\frac{T}{\gamma(\gamma-1) \mathrm{M}^{2}}+\frac{1}{2} \mathbf{v}^{T} \mathbf{v},
$$

where $M$ is the reference Mach number, $\mathbf{v}$ is the velocity vector, and $\gamma$ is the ratio of specific heats. The spatial domain occupied by the fluid is $\Omega=\left\{\mathrm{x} \in \mathbb{R}^{2}: x_{2}>0\right\}$, $\Gamma_{c}=\left\{\mathbf{x} \in \mathbb{R}^{2}: x_{2}=0, a \leq x_{1} \leq b\right\}$ is the portion of the solid boundary on which control is actuated, and $\left(t_{0}, t_{f}\right)$ is the time period of interest. The function $g=-v_{2}$ in the boundary conditions, $\mathbf{B}(\mathbf{u}, \nabla \mathbf{u}, g)=\mathbf{0}$, is the transpiration boundary control that is actuated on the controlled boundary $\Gamma_{c}$. Positive $g$ denotes suction while negative $g$ corresponds to injecting fluid into the domain $\Omega$.

\section{Wall boundary conditions}

Transpiration boundary control $g$ takes place in the near field region where nonlinearities, unsteadiness, and spatial gradients are often significant. Not surprisingly, implementation of a boundary treatment that can accommodate this control mechanism is not an easy task. Moreover, the hyperbolic nature of the Euler equations (and its discretization) often leads to the propagation of nonphysical error waves associated with inappropriately imposed and/or implemented boundary conditions. This can ultimately contaminate the solution everywhere in the domain. This difficulty is particularly acute for high-order central difference discretizations when mated with explicit timeadvancement schemes since the discrete dispersion relation permits highly oscillatory numerical error waves that can propagate faster than the physical waves of the system often leading to restrictive time-step constraints.

The implementation of slip-wall boundary conditions (zero normal velocity) for aeroacoustic computations has received attention, most notably the ghost cell method of Tam and Dong ${ }^{4}$ and Kurbatski and Tam. ${ }^{5}$ But, implementation of transpiration type boundary conditions for inviscid compressible flows has not been received much attention. We view the transpiration boundary condition as an inflow/outflow boundary condition where the number of physical boundary conditions is dictated by the characteristic speeds associated with the unit outward normal $\mathbf{n}$. These speeds are $\mathbf{v}^{T} \mathbf{n}, \mathbf{v}^{T} \mathbf{n}$ and $\mathbf{v}^{T} \mathbf{n} \pm c$ where $\mathbf{v}$ and $c$ are the local velocity and sound-speed, respectively. When one of these characteristic speeds is negative, the associated characteristics propagates from outside the boundary towards the interior of the domain and a physical boundary condition must be imposed. On the other hand, a positive speed indicates flow of information from the inner domain towards the boundary necessitating the imposition of 
a numerical boundary condition. Therefore, one physical and three numerical boundary conditions have to imposed for subsonic suction and three physical and one numerical boundary conditions are needed for subsonic blowing. Hence, for an impermeable wall or suction, $\mathbf{v}^{T} \mathbf{n} \geq 0$, the following physical boundary condition must be satisfied:

$$
\mathbf{v}^{T} \mathbf{n}-g=0
$$

However, when fluid is injected into the domain, not only must the wall normal velocity be specified, but, as suggested by the characteristic speeds normal to the boundary, two other flow quantities must be imposed. The appropriate selection of the additional flow quantities that should be constrained can impact both the implementation as well as the accuracy of the final method. Based on extensive experimentation, we have selected entropy and vorticity as the additional flow quantities that are specified in our transpiration boundary condition. This was motivated by the well-known fact that entropy and vorticity information propagates along the incoming characteristics and, as shown below, we were able to implement these boundary conditions within our high-order finite difference discretization in such a way that high-accuracy and numerical stability were retained. In summary, the following physical boundary conditions are imposed for $\mathbf{v}^{T} \mathbf{n}<0$ :

$$
\begin{aligned}
\mathbf{v}^{T} \mathbf{n} & =g \\
\ln \left(\frac{T^{1 /(\gamma-1)}}{\rho}\right) & =S_{0} \\
\partial_{x_{2}} v_{1}-\partial_{x_{1}} v_{2} & =\omega_{0}
\end{aligned}
$$

In this study, we require the injected fluid be isentropic and irrotational by setting $\omega_{0}=S_{0}=0$. Injection of rotational fluid will be explored in future work. Although we have used these boundary conditions successfully in several optimal control applications, mathematical well-posedness of the compressible Euler equations with these boundary conditions has not been established and success of these boundary conditions may be dependent on their implementation, which is discussed below.

\section{Optimal control problem}

In this paper, we seek to minimize the following objective function:

$$
\begin{aligned}
& J(g)=\frac{1}{2} \int_{t_{0}}^{t_{f}} \int_{\Omega_{\mathrm{obs}}} \alpha_{0}\left(p-p_{a}\right)^{2} d \mathbf{x} d t+ \\
& \frac{1}{2} \int_{t_{0}}^{t_{f}} \int_{\Gamma_{c}}\left(\alpha_{1} g_{t}^{2}+\alpha_{2} g^{2}+\alpha_{3} g_{x_{1}}^{2}+\alpha_{4} g_{x_{1} x_{1}}^{2}\right) d x_{1} d t
\end{aligned}
$$

The first term is the square of the acoustic amplitude integrated over the observation region $\Omega_{\text {obs }}$ and time horizon $\left[t_{0}, t_{f}\right]$ where $p_{a}$ is the ambient or the steady mean-flow pressure distribution. The second term is a regularization term that enforces certain smoothness requirements on the controls. See Ref. 2 for more details. In addition we require that the control $g$ is zero at the initial time $t_{0}$ to ensure compatibility between the initial flow field and boundary data and we impose that $g$ and its slope $g_{x_{1}}$ vanish at the endpoints $x_{1}=a$ and $x_{1}=b$. In (5) $\alpha_{0}, \ldots, \alpha_{4}$ are positive weighting parameters.

\section{Adjoint equations}

We use a gradient-based optimization procedure to solve this optimal control. The gradient is computed with the continuous adjoint method. As mentioned before, we are guided by the optimize-then-discretize approach. The details for the derivation of the adjoint partial differential equations and adjoint boundary conditions will be presented in another paper. Because of space limitations, we state only the final form.

The adjoint variables $\boldsymbol{\lambda}$ to the flow equations (1) satisfy the differential equation

$$
\mathbf{M}^{T} \boldsymbol{\lambda}_{t}+\sum_{i} \mathbf{A}^{i^{T}} \boldsymbol{\lambda}_{x_{i}}=\mathbf{r}
$$

where $\mathbf{M}=\mathbf{q}_{\mathbf{u}}(\mathbf{u})$ and $\mathbf{A}^{i}=\mathbf{F}_{\mathbf{u}}^{i}(\mathbf{u})$. The source term $\mathbf{r}$ in (6) is obtained by differentiating the right hand side in (5) with respect to the primitive variables. It is identically zero outside the observation region $\Omega_{\text {obs }}$ and

$$
\mathbf{r}=\frac{p-p_{a}}{\gamma \mathrm{M}^{2}}\left(\begin{array}{c}
T \\
0 \\
0 \\
\rho
\end{array}\right)
$$

inside $\Omega_{\text {obs }}$. The final time condition for the adjoint variables is given by

$$
\lambda\left(t_{f}, \mathbf{x}\right)=0 .
$$

Since the boundary conditions $\mathbf{B}(\mathbf{u}, \nabla \mathbf{u}, g)=\mathbf{0}$ depend on the sign of the normal velocity, the boundary conditions for the adjoint equation also depend on $-v_{2}=\mathbf{v}^{T} \mathbf{n}$. The Euler equations with boundary conditions imply the following boundary conditions for the adjoint.

For suction ( $\left.g=-v_{2} \geq 0\right)$ we obtain the three boundary conditions

$$
\begin{aligned}
\lambda_{3}+\frac{\gamma}{\gamma-1} g \lambda_{4} & =0 \\
g\left(\lambda_{2}+v_{1} \lambda_{4}\right) & =0 \\
g\left(\lambda_{1}-\lambda_{4}\left(\frac{1}{2} v_{1}^{2}+\frac{\gamma+1}{2(\gamma-1)} g^{2}\right)\right) & =0 .
\end{aligned}
$$

The solid surface is a special case of suction with $g=0$ and it can be seen from (9) that only one boundary condition

$$
\lambda_{3}=0
$$

is required. For blowing $\left(g=-v_{2}<0\right)$, we obtain two boundary conditions

$$
\begin{aligned}
g\left(\lambda_{2}+v_{1} \lambda_{4}\right) & =0 \\
\left(1+g^{2} \frac{M^{2}}{T}\right)\left(\lambda_{3}+\frac{\gamma}{\gamma-1} g \lambda_{4}\right) & + \\
g \frac{M^{2}}{T}\left(\lambda_{1}-\lambda_{4}\left(\frac{1}{2} v_{1}^{2}+\frac{\gamma+1}{2(\gamma-1)} g^{2}\right)\right) & =0 .
\end{aligned}
$$


Looking at the adjoint equation, (6), one would expect only one boundary condition in the case $g=-v_{2}<0$. A full analysis of these adjoint boundary conditions is in progress. However, it should be noted that the derived boundary conditions for suction and blowing are compatible as $g \rightarrow 0$.

\section{Implementation}

In this section, we focus on the discretization scheme and the implementation of the transpiration boundary condition for the state equations.

\section{Discretization}

Numerical simulation of aeroacoustic phenomena demands high numerical accuracy (i.e. low dissipation and dispersion) to accurately resolve convective flow-features over a wide range of space/time scales and amplitudes. To meet this need, most prior approaches have utilized high-order accurate finite difference methods such as the compact schemes ${ }^{6}$ and the dispersion relation preserving methods. ${ }^{7}$ Our study focuses on the application of optimal control to aeroacoustic problems and for this purpose, we have initially chosen to use a traditional central finitedifference method with explicit high-wavenumber damping and simple boundary treatments. Such a method allows us to explore the issues involved in applying optimal control theory to aeroacoustic flows while avoiding complications that may arise due to more complex discretizations.

With this background, our Euler flow solver is based on a conservative extension of the explicit finite-difference method described in Ref. 8. The Euler equations are formulated in a generalized coordinate system where the physical domain is mapped to a computational space; a unit square divided into an equally spaced grid system. This transformation allows clustering of grid points in regions of high gradients, simplifies the implementation of the boundary conditions, and allows the code to be used for moderately complex geometries. While the code supports optimized finite-differences with up to seven point stencils, for this study spatial derivatives are approximated using standard sixth-order accurate central differences in the interior with third-order biased and one-sided differences used at boundaries that are designed to enhance stability when used with explicit time advancement methods. ${ }^{9}$ To suppress the growth of high-wavenumber error modes, a fourth-order artificial dissipation term is added to the right hand side of the discretized equations. This dissipation term is computed using fourth-order accurate finite-differences and the dissipation parameter, $\epsilon$, is chosen to damp out the error modes while avoiding excessive dissipation in the resolved scales as established through numerical experimentation. Sponge terms $^{8,10}$ are used in the vicinity of the farfield boundary with a one-dimensional Riemann invariant treatment at the farfield boundary. ${ }^{8}$

\section{Implementation of wall transpiration boundary condition}

Our implementation of nearfield inflow/outflow boundary condition is based on the approach originally formulated by J. Sesterhenn. ${ }^{1}$ Sesterhenn expresses the invis-

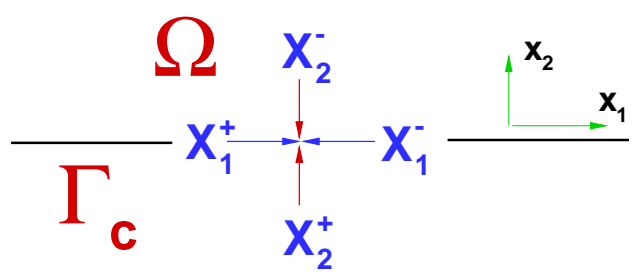

Fig. 1 Pseudo-waves at a subsonic boundary point.

cid part of the equations as a decomposition into several plane waves aligned with the numerical grid in an attempt to merge finite differencing with schemes based on compatibility equations such as Moretti's $\lambda$-scheme. ${ }^{11}$ In this approach, transport equations are written for pressure, normal and tangential velocities in terms of the 'pseudo' acoustic wave amplitudes. These 'pseudo' waves may not have any physical significance in two or three dimensional flows but in one dimension coincide with the temporal change of acoustic wave amplitudes closely related with the Riemann invariants of homentropic flows. Also, the introduction of these pseudo-waves allows a more direct and natural implementation of wall boundary conditions as opposed to the locally one-dimensional inviscid approximation of Poinsot and Lele. ${ }^{6}$ We use Sesterhenn's decomposition only on the wall boundary to implement the transpiration boundary conditions (3) and (4).

We define the following 'pseudo-waves' in the $x_{1}$ and $x_{2}$ directions

$$
\begin{aligned}
& X_{1}^{ \pm}=\left(v_{1} \pm c\right)\left(\frac{1}{\rho c} \partial_{x_{1}} p \pm \partial_{x_{1}} v_{1}\right) \\
& X_{2}^{ \pm}=\left(v_{2} \pm c\right)\left(\frac{1}{\rho c} \partial_{x_{2}} p \pm \partial_{x_{2}} v_{2}\right)
\end{aligned}
$$

Transport equations for the velocity components and pressure can be recast in terms of these pseudo-waves as

$$
\begin{aligned}
\partial_{t} v_{2}= & -\frac{1}{2}\left(X_{2}^{+}-X_{2}^{-}\right)-v_{1} \partial_{x_{1}} v_{2}, \\
\partial_{t} v_{1}= & -\frac{1}{2}\left(X_{1}^{+}-X_{1}^{-}\right)-v_{2} \partial_{x_{2}} v_{1}, \\
\partial_{t} p= & -\frac{\rho c}{2}\left(X_{1}^{+}+X_{1}^{-}+X_{2}^{+}+X_{2}^{-}\right) .
\end{aligned}
$$

At boundary points $\left(x_{1}, 0\right), X_{1}^{ \pm}$are determined from the boundary data available at the current time. However, in the $x_{2}$ direction, the Mach number $M_{2}=v_{2} / c$ determines where the $X_{2}$ pseudo-waves are coming from. For instance, for locally subsonic flow, $X_{2}^{+}$enters the domain from outside while $X_{2}^{-}$leaves the domain, as seen in Fig. 1. Waves which enter the domain are specified so that the imposed physical boundary conditions are satisfied. As mentioned earlier, one physical boundary condition, $v_{2}=-g$, has to be imposed on the boundary for subsonic suction. The unknown pseudo-wave $X_{2}^{+}$is determined from (13a).

$$
X_{2}^{+}=X_{2}^{-}+2\left(\partial_{t} g+v_{1} \partial_{x_{1}} g\right)
$$

The time derivative of pressure can now be computed by substituting for $X_{2}^{+}$from (14) in (13c). The energy equation can be written in terms of entropy as:

$$
\partial_{t} S+v_{1} \partial_{x_{1}} S+v_{2} \partial_{x_{2}} S=0
$$


where $\partial_{x_{2}} S$ is computed from the interior domain using a one-sided finite-difference stencil allowing us to compute the time derivative of entropy from (15). Similarly, if $\partial_{x_{2}} v_{1}$ is computed by a one-sided finite-difference stencil, the time derivative of $v_{1}$ can also be computed from (13b). Now, we have $\partial_{t} v_{1}, \partial_{t} v_{2}, \partial_{t} p$, and $\partial_{t} S$ at our disposal and can easily compute the temporal derivative of any other flow quantity. For instance, to compute $\partial_{t} \rho$, one can use the enthalpy equation

$$
d h=T d s+\frac{d p}{\rho}
$$

in conjunction with the equation of state $\mathrm{Eq}$ (2) to write the density time derivative in terms of entropy and pressure.

For blowing, however, three physical boundary conditions have to be imposed, $v_{2}=-g, S=0$ and $\omega=0$ where $S$ and $\omega$ denote entropy and vorticity, respectively. Again, the unknown pseudo-wave is determined from (14) and the pressure time derivative is subsequently computed from (13c). The time derivative of density is found by enforcing zero rate of change in entropy in (16):

$$
\partial_{t} \rho=\frac{1}{c^{2}} \partial_{t} p
$$

Vorticity $\omega$ is given by

$$
\omega=\partial_{x_{2}} v_{1}-\partial_{x_{1}} v_{2}
$$

where $\partial_{x_{1}} v_{2}=-\partial_{x_{1}} g$ is a known quantity. This boundary condition is enforced, weakly, by substituting $\partial_{x_{2}} v_{1}=$ $\partial_{x_{1}} v_{2}$ in the RHS of (13b).

\section{Implementation of adjoint boundary conditions}

To exercise the flexibility of the optimize-then-discretize approach, our adjoint boundary conditions are implemented based on characteristic variables and not by adapting the approach used for the state boundary conditions. More details will be given in a forthcomming paper.

At the farfield boundaries we use sponges to reduce reflections as outgoing waves leave the computational domain. The sponge term used in the adjoint is identical to what one would obtain by including the sponge term for the state in the adjoint computations.

\section{Numerical Results}

\section{Test case 1}

In this section we present optimal control results for the test problem depicted in Fig. 2. In the following, the source period $T_{p}$ and the wave length $L$ are used for nondimensionalization. A time harmonic line source is located at a distance $H=5$ from a solid wall. The computational domain is $\Omega=[-3.5,3.5] \times[0,7]$ with periodic boundary conditions in the horizontal direction and a sponge-type non-reflecting far-field boundary condition enforced at the top boundary. The control objective is the integral of the square of the pressure deviation from the ambient pressure over the observation region, $\Omega_{\mathrm{obs}}=[-2,2] \times[1 / 2,3 / 2]$

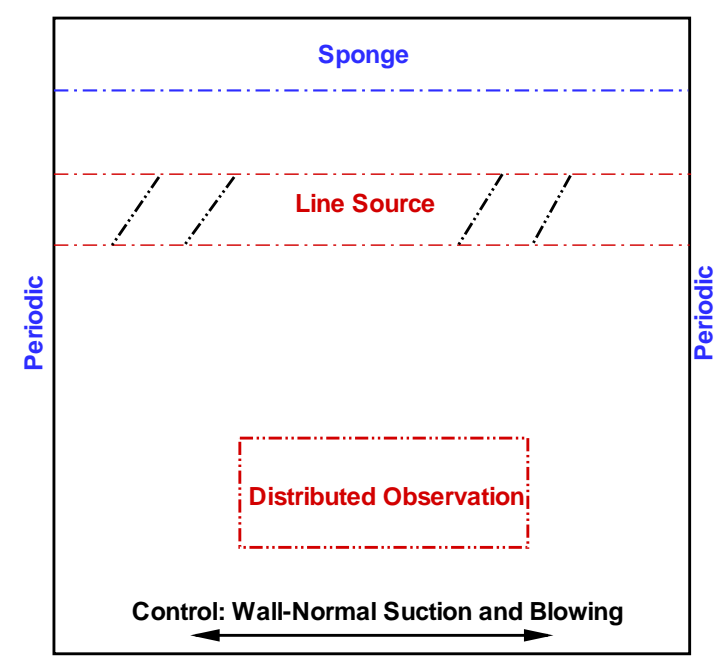

Fig. 2 A schematic diagram of test case 1

(the rectangular box shown in each of the contour plots of Figs. 3, 4, 6b) ) from time $t_{0}=30$ to $t_{f}=50$. As the pressure fluctuations are usually very small, the weigh $\alpha_{0}$ in the control objective (5) is chosen to be large relative to the other weights. We use $\alpha_{0}=10^{6}, \alpha_{1}=10^{-3}, \alpha_{2}=10^{-7}$, $\alpha_{3}=\alpha_{4}=10^{-3}$. Wall-normal transpiration constitutes the boundary control, defined over $[-3,3] \times\left[t_{0}, t_{f}\right]$. The time interval is comprised of 800 uniform time-steps of size $\Delta t=0.025$ and the spatial mesh $(141 \times 141)$ has uniform spacing of $\Delta x=\Delta y=0.05$. As the line source is harmonic and the effect of nonlinearities away from the source region is negligible, the flow field exhibits a limit-cycle behavior with period $T_{p}$ as $t \rightarrow \infty$. The initial time $t_{0}$ is chosen large enough for this limit-cycle pattern to be effectively established in the domain. Figure 3 shows contours of pressure fluctuations about the ambient pressure, $p-p_{\mathrm{a}}$, for the uncontrolled flow within one period of oscillation.

The analytical solution for the infinite dimensional nocontrol problem (in terms of dimensional quantities) can be easily found by superimposing an image line source at $-5 L$. For instance, the second component of velocity, $v_{2}$, and pressure, $p$, can be written as,

$$
\begin{gathered}
v_{2}=R e\left(v_{m} e^{2 \pi i\left(\frac{H+x_{2}}{L}-\frac{t}{T_{p}}\right)}-v_{m} e^{2 \pi i\left(\frac{H-x_{2}}{L}-\frac{t}{T_{p}}\right)}\right), \\
p=\operatorname{Re}\left(p_{m} e^{2 \pi i\left(\frac{H+x_{2}}{L}-\frac{t}{T_{p}}\right)}+p_{m} e^{2 \pi i\left(\frac{H-x_{2}}{L}-\frac{t}{T_{p}}\right)}\right),
\end{gathered}
$$

where $R e$ denotes the real part of the complex argument and $v_{m}$ and $p_{m}$ are the velocity and pressure amplitude of the harmonic line source, respectively. Equation (19) can be simplified further:

$$
\begin{aligned}
v_{2} & =4 v_{m} \sin \left(\frac{2 \pi x_{2}}{L}\right) \cos \left(2 \pi\left(\frac{H}{L}-\frac{t}{T_{p}}+\frac{1}{4}\right)\right)(20 \mathrm{a}) \\
p & =4 p_{m} \cos \left(\frac{2 \pi x_{2}}{L}\right) \cos \left(2 \pi\left(\frac{H}{L}-\frac{t}{T_{p}}\right)\right) \cdot \quad(20 \mathrm{~b})
\end{aligned}
$$



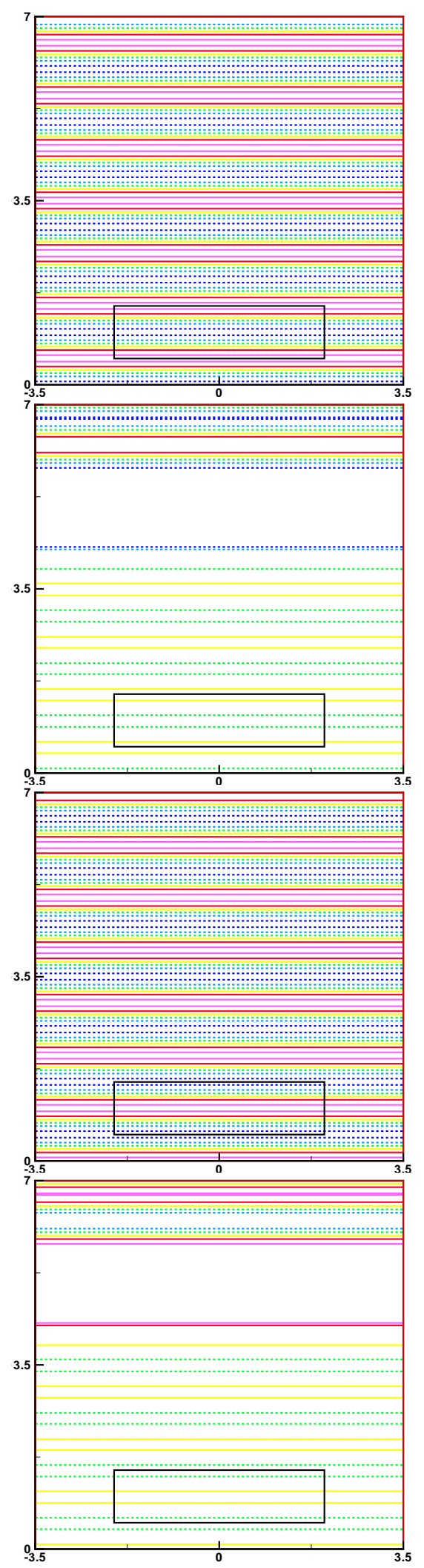

Fig. 3 Contours of $p-p_{\mathrm{a}}$ for the no-control case at four equally spaced instants spanning one period of oscillation $T_{p}$. ( 6 equally spaced contour levels between $\pm 7.0 \times 10^{-6}$ )

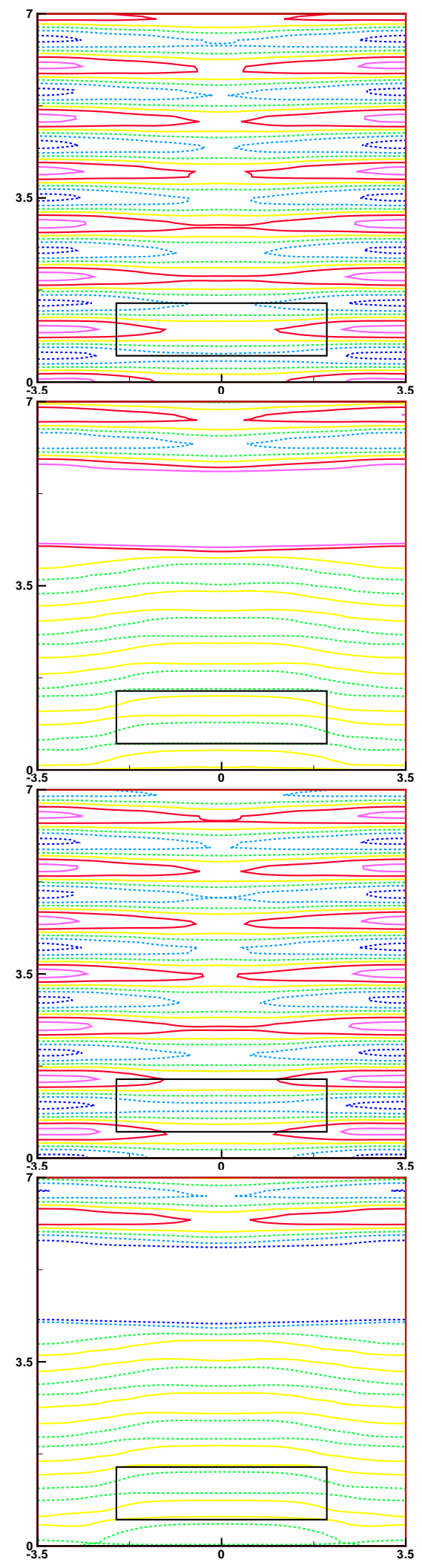

Fig. 4 Contours of $p-p_{\text {a }}$ for the optimal control case at four equally spaced instants spanning one period of oscillation $T_{p}$. ( 6 equally spaced contour levels between $\pm 7.0 \times 10^{-6}$ ) 


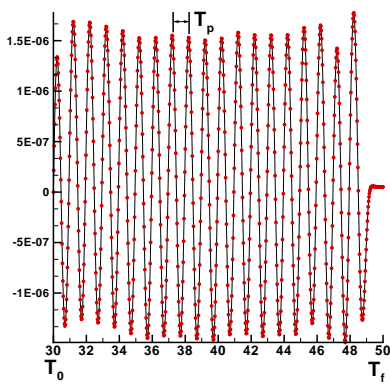

a)

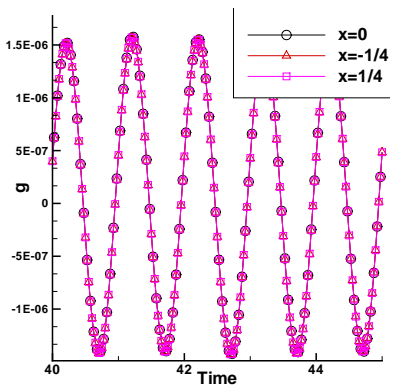

b)

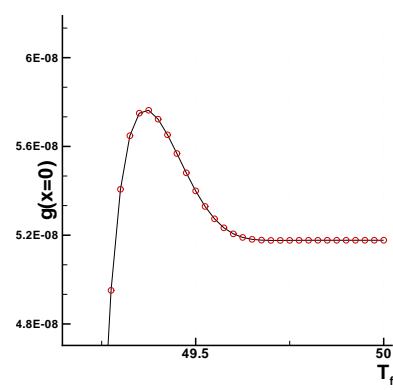

a)

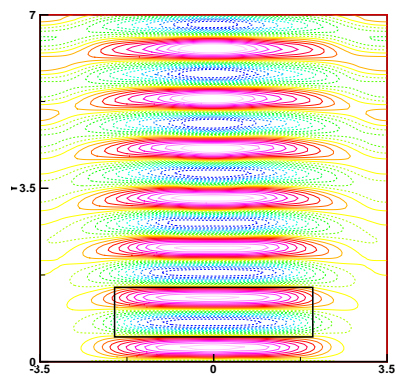

b)
Fig. 5 a) Time history of control g at $x=0$. b) Time history of control g at $x=0, \pm 1 / 4$.

Equation (20a) satisfies the inviscid wall boundary condition at $x_{2}=0$ at all times and further examination of (20b) shows that for $t=H / c+T_{p}(2 n+1) / 4, n=0,1,2,3, \ldots$, the pressure fluctuation diminishes everywhere in the domain. This behavior is seen in the second and fourth plots of Fig. 3.

The initial value of the control objective is $1.2 \times 10^{-3}$. After 15 nonlinear conjugate gradient iterations, ${ }^{12}$ the control objective is reduced to $6.6 \times 10^{-4}$ which is about $45 \%$ of its initial value. The acoustic pressure contours for the controlled run are shown in Fig. 4. In order to analyze the behavior of the computed control, the time history of the control at $x=0, \pm 1 / 4$ is plotted in Fig. 5. Figure 5(a) depicts the time history of control at $x=0$. It can be seen that the control has no difficulty in picking up the source frequency and it oscillates with an approximately constant amplitude for most of the time window $\left(t_{0}, t_{f}\right)$. Close to the final time it loses the harmonic behavior and becomes approximately constant. Figure 5(b) shows the time history of control at three locations on the wall, $x=0, \pm 1 / 4$, separated by a quarter wave length. This plot demonstrates that the control at these three locations are exactly in phase. Further checks reveal that the control indeed is constant across the control region and is only a function of time. Because the lower edge of the observation region is located at a distance $1 / 2$ above the wall, it takes about $1 / 2$ time units for the effect of the boundary actuation to be felt in the observation region - explaining the behavior of control near the final time shown in Fig. 6(a). From $t=49.5$ onward, the control cannot affect the observation term and, hence, it tries to minimize the contribution of the regularization term. The contribution of the spatial derivatives $\mathbf{g}_{x_{1}}$ and $\mathbf{g}_{x_{1} x_{1}}$ to (5) is zero and the contribution of the time regularization term $\left\|\mathbf{g}_{t}\right\|_{2}^{2}$ is approximately $4 \pi^{2} \approx 39.5$ times larger than the regularization term $\|\mathbf{g}\|_{2}^{2}$ for a simple harmonic oscillation. Therefore, the main focus of the optimal control in the time period $\left(39.5, t_{f}\right)$ is to initially reduce $\mathrm{g}_{t}$, which is clearly achieved by creating a plateau near the final time $t_{f}$. To understand the behavior of the computed control, one can subtract the uncontrolled pressure field from the optimal control pressure field as shown in Fig. 6(b). As the amplitude of flow quantities are small, the effect of the nonlinear terms in the governing equations
Fig. 6 a) Behavior of control g near the final time $t_{f}$, b) Contours of $p_{\text {optimal }}-p_{\text {nocontrol at time } t=40.5 \text { isolating the }}$ effect of boundary control actuation. (20 evenly spaced contours between $\pm 3.5 \times 10^{-6}$ )

is negligible and the contour plot of Fig. 6(b) isolates the effect of boundary actuation accurately. It is clearly seen that the boundary control creates a nearly planar wave to counter the wave system of (20) in the observation region. A perfect cancellation is not possible because the wave produced by the control cannot cancel the wave system of (20) at all times. The optimal control targets instants of time at which the observation region has high amplitude waves (the first and third snapshots of Fig. 3 and tries to reduce it by producing the wave shown in Fig. 6(b). In so doing, it disturbs the approximatly silent instants observed in the second and fourth plots of Fig. 3.

\section{Test case 2}

In this test case, we control the scattered and refracted wave pattern arising from the interaction of a point sound source with an inviscid vortex. Again, all flow quantities are nondimensionalized with source period $T_{p}$ and wave length $L$. A monopole sound source, modeled as a source term in the energy equation, is located at $(0,5)$ and interacts with an inviscid vortex ${ }^{13}$ of circulation $2 \pi / 5$ (counter-clockwise) and radius $1 / 2$ located at $(0,3.5)$. The computational domain, control objective, and the spatial and temporal discretizations are identical to that of test case 1. Sponge-type nonreflecting far-field boundary conditions are now used on the left, right and top boundaries. The distance between the vortex and the solid wall is large enough to comfortably ignore the effect of the image vortex in the optimization time interval. Hence, the vortex is considered to be stationary and the mean flow pressure distribution to be steady. The time interval is comprised of 600 uniform time-steps of size $\Delta t=0.025$ from time $t_{0}=30$ to time $t_{f}=45$. Again, the initial time is larger than the time required for the limit-cycle pattern to be effectively established in the domain.

After 20 optimization iterations the control objective is reduced from 0.49 to 0.34 . The acoustic pressure contours for the no control and optimal control simulations are shown in Fig. 7(a)-(b) respectively. In the no control simulation, the scattered wave behind the vortex is mostly seen in the right half of the observation region, which is due to the counter-clockwise circulation of the vortex, and the left 

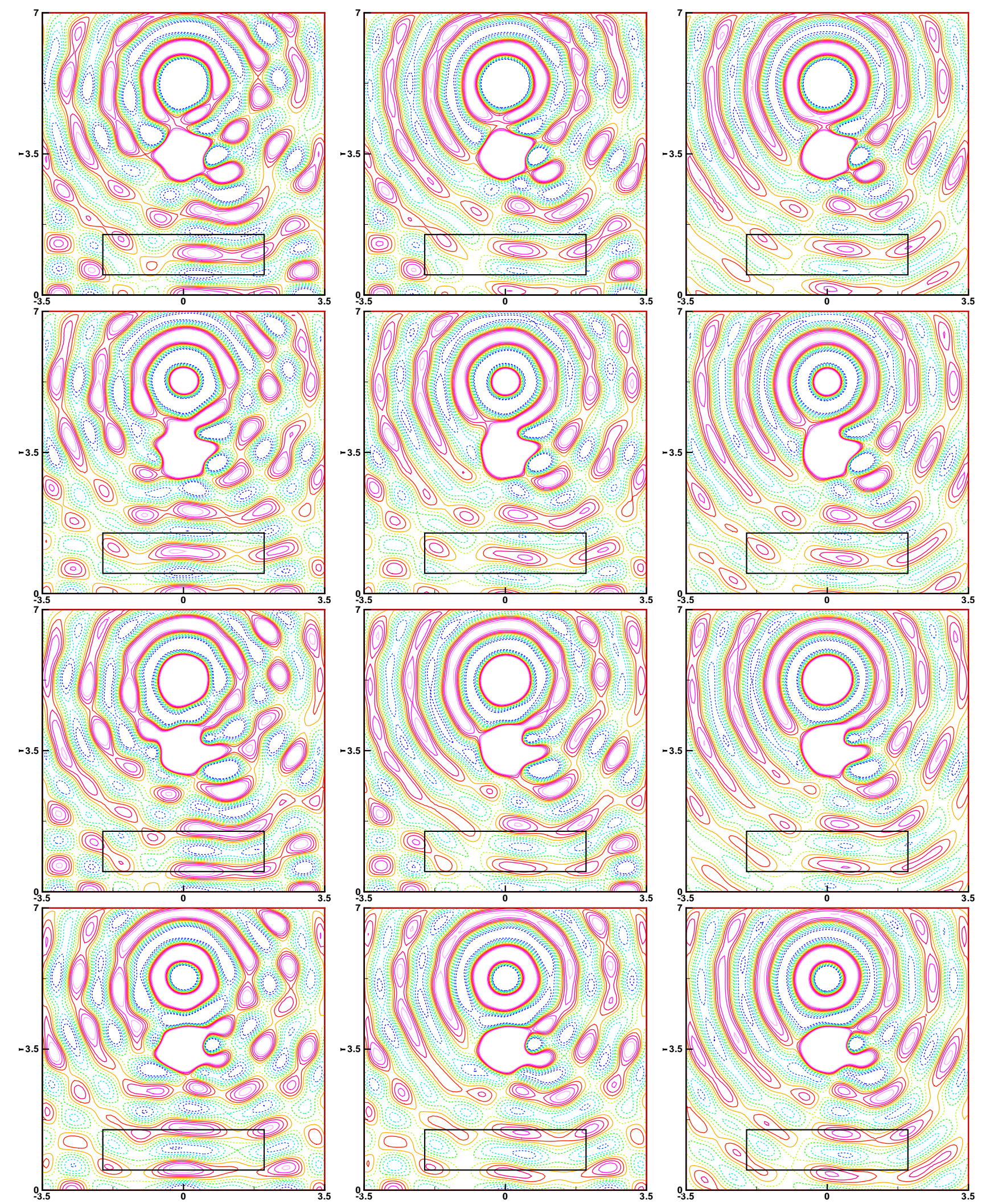

(a) No control

(b) Optimal control

(c) Far-field BC on bottom boundary

Fig. 7 Contours of $p-p_{\mathrm{a}}$ at four equally spaced instants spanning one period of motion $T_{p}$. (6 contour levels between $\pm 1.75 \times$ $\left.10^{-4}\right)$ 


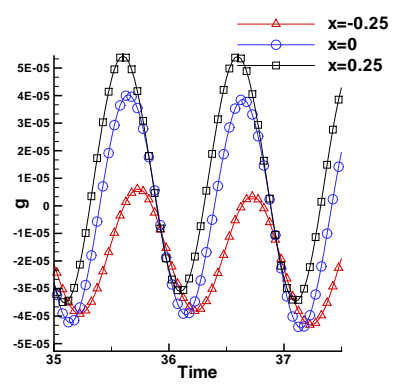

a) b)

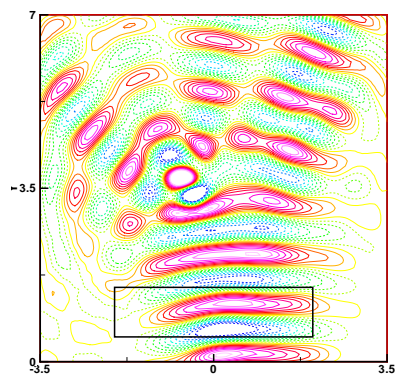

Fig. 8 a) Time history of control $\mathbf{g}$ at $x=0, \pm 1 / 4$, b) Contours of $p_{\text {optimal }}-p_{\text {nocontrol }}$ at time 40.75 isolating the effect of boundary control actuation. ( 20 contours between $\pm 9.0 \times 10^{-5}$ )

half is relatively silent. The solid wall intensifies the incident waves and forces the waves to move horizontally. Comparing the optimal control snapshots with the corresponding no control plots reveals that the optimal control targets the reflection off the solid wall deemed responsible for the intensification of the scattered and refracted waves. The computed control makes the wall transparent to the incident waves and prevents them from reflecting back into the observation region. To verify this conjecture, a no-control simulation is performed where the solid wall is replaced with a nonreflecting boundary condition based on Riemann extrapolation. Figure 7(c) shows four snapshots of the acoustic pressure field at the same instants of time. It is evident that the control actuation in the right half of the observation region mimics an absorbing type boundary condition. This observation demonstrates the flexibility of the proposed transpiration boundary conditions.

In the no-control run, the refracted/reflected wave pattern seen in the middle of the observational region is approximately horizontal. But, the wave system produced by the boundary actuation (Fig. 8(b)) and the wave pattern observed in the center of the optimal control plots (Fig. 7) slant in opposite directions. The production of the slanted wave of Fig. 8(b) by the boundary actuation is further validated by looking at Fig. 8(a) where the time history of control at three positions, $x=-0.25,0,0.25$, separated by quarter wave length, are plotted. The phase difference in control actuation at these three locations allows the production of waves with slanted fronts. It is interesting to note that the control amplitude is smaller at $x=-0.25$ than the other two locations. This shows that the optimal control tries to avoid disturbing the relatively silent left half of the observation region while attenuating the noisy right half of this region.

\section{Conclusions}

This work focuses on some important issues encountered in the optimal boundary control of aeroacoustic flows governed by high-order central finite difference discretizations of the unsteady Euler equations. The control of acoustic waves, which are typically four orders of magnitude smaller than energetically dominant flow quantities, requires extreme care at the numerical modeling stage. Proper resolution of fluctuating flow quantities, nonreflecting farfield boundary treatments, and solid wall modeling are issues of great importance in computational aeroacoustics and have been studied extensively in the literature. The optimal control of aeroacoustic waves with transpiration boundary control also requires the formulation and implementation of an accurate near field inflow/outflow type boundary condition on the controlled segment of the boundary. It is argued, based on the characteristic wave propagation speeds normal to the controlled boundary, that subsonic suction requires one while blowing requires three physical boundary conditions. The transpiration velocity is one of the imposed boundary conditions for both suction and blowing, while we also require that injected fluid also be irrotational and isentropic. The implementation of the transpiration boundary condition is based on a decomposition of the inviscid fluxes into several planar pseudo-waves aligned with the boundary tangent and boundary normal direction using a technique originally introduced by Sesterhenn. ${ }^{1}$ The transpiration boundary condition is tested with two optimal control test problems. A continuous adjoint gradient-based method is used to solve the optimization problems and the adjoint equation, its end time condition and boundary conditions are stated and discussed. Despite the difference in the number of derived adjoint boundary conditions for suction, blowing, and solid wall; the boundary conditions are compatible as the control tends to zero. The first test problem demonstrates that the transpiration control actuation is capable of producing well-resolved acoustic waves that reduce the observed sound amplitude by means of wave-cancellation. In the second test problem, the transpiration boundary condition mimics a nonreflecting boundary condition on the controlled surface in order to eliminate the intensifying effect of reflections off the solid wall. The success of the transpiration control actuation for these test problems has emboldened us to investigate the feasibility of this control actuation for controlling the impulsive noise associated with the Blade-Vortex Interaction (BVI) phenomenon in our future work.

\section{Acknowledgments}

This work was supported by Texas ATP grant 003604 0001, 1999, and in part by the Los Alamos National Laboratory Computer Science Institute (LACSI) through LANL contract number 03891-99-23, as part of the prime contract (W-7405-ENG-36) between the Department of Energy and the Regents of the University of California. Computations were performed on an SGI Origin 2000 which was purchased with the aid of NSF SCREMS grant 98-72009.

\section{References}

${ }^{1}$ Sesterhenn, J., "A Characteristic-Type Formulation of the NavierStokes Equations for High Order Upwind Schemes," Computers \& Fluids, Vol. 30, 2001, pp. 37-67.

${ }^{2}$ Collis, S. S., Ghayour, K., Heinkenschloss, M., Ulbrich, M., and Ulbrich, S., "Towards Adjoint-Based Methods for Aeroacoustic Control," AIAA Paper 2001-0821, 2001. 
${ }^{3}$ Collis, S. S., Ghayour, K., Heinkenschloss, M., Ulbrich, M., and Ulbrich, S., "Numerical Solution of Optimal Control Problems by the Compressible Navier-Stokes Equations," Proceedings of the International Conference on Optimal Control of Complex Structures, edited by G. Leugering, J. Sprekels, and F. Tröltzsch, Birkhäuser Verlag, 2001.

${ }^{4}$ Tam, C. K. W. and Dong, Z., "Wall Boundary Conditions for HighOrder Finite Difference Schemes in Computational Aeroacoustics," Theoretical and Computational Fluid Dynamics, Vol. 6, No. 6, 1994, pp. 303322.

${ }^{5}$ Kurbatskii, K. A. and Tam, C. K. W., "Cartesian Boundary Treatment of Curved Walls for High-Order Computational Aeroacoustics Schemes," AIAA J., Vol. 35, No. 1, 1997, pp. 133-140.

${ }^{6}$ Poinsot, T. J. and Lele, S. K., "Boundary Conditions for Direct Simulations of Compressible Viscous Flows," J. Comp. Phys., Vol. 101, No. 1, 1992, pp. 104-129.

${ }^{7}$ Tam, C. K. W. and Webb, J. C., "Dispersion-Relation-Preserving Finite Difference Schemes for Computational Acoustics," J. Comp. Phys., Vol. 107, 1993, pp. 262-281.

${ }^{8}$ Collis, S. S., A Computational Investigation of Receptivity in HighSpeed Flow Near a Swept Leading-Edge, Ph.D. thesis, Stanford University, March 1997.

${ }^{9}$ Carpenter, M. H., Gottlieb, D., and Abarbanel, S., "Stable and Accurate Boundary Treatments for Compact, High-Order Finite Difference Schemes," Appl. Num. Math., Vol. 12, No. 1-3, 1993, pp. 55-87.

${ }^{10}$ Israeli, M. and Orszag, S. A., "Approximation of Radiation Boundary Condition," Journal of Computational Physics, Vol. 41, 1981, pp. 115-135.

${ }^{11}$ Moretti, G., "The $\lambda$-scheme," Computers \& Fluids, Vol. 7, 1979, pp. 191-205.

${ }^{12}$ Nocedal, J. and Wright, S. J., Numerical Optimization, Sringer Verlag, Berlin, Heidelberg, New York, 1999.

${ }^{13}$ Shu, C.-W., "Esentially Non-Oscillatory and Weighted Essentially Non-Oscillatory Schemes for Hyperbolic Conservation Laws," Tech. Rep. 97-65, ICASE, November 1997. 